\title{
A Method for Calculating Solutions of Parabolic Equations with a Free Boundary
}

\author{
By Milton E. Rose
}

1. Introduction. The description of phenomena involving phase transitions leads to a class of parabolic partial differential equations with a free interior boundary which marks the interface separating the phases. The simplest nonlinear problem of this type was first treated by J. Stefan in 1891 and considerable attention has been focused on such problems in recent years [4], [6], [9].

A typical mathematical formulation of this type of problem is the following:

Problem I: Determine functions $x(t)$ and $u(x, t)$ satisfying

$$
\begin{aligned}
& \text { (a) } u_{x x}(x, t)=u_{t}(x, t), \quad 0<x<x(t), \quad t>0 \\
& \text { (b) } u_{x}(0, t)=-1, \quad t>0 \\
& \text { (c) } \dot{x}(t)=-u_{x}(x(t), t), \quad t>0 \\
& \text { (d) } u(x, t)=0, \quad x \geqq x(t), \quad t \geqq 0 \\
& \text { (e) } x(0)=0 \text {. }
\end{aligned}
$$

With suitable choice of units this formulation describes, for example, the recrystallization due to uniform heating at one end of a semi-infinite substance which is initially at its critical temperature [3]. Equations (1c), (1d), and (1e) serve to describe the interface separating the phases of crystalline state. Effective numerical procedures and proofs of their validity have been described by Douglas and Gallie [2], and Trench [8].

The object of this paper is to suggest a computational approach to general problems of this type which has the feature that the path of the interface is not regarded as an explicitly. imposed interior boundary condition. The method is closely related to a proposal by P. Lax [7] for calculating weak solutions of hyperbolic equations in conservation form as the limits of suitable finite difference equations. Several problems involving shocks and contact discontinuities have been successfully treated by Lax from this point of view. The present investigation was motivated by the question of whether or not phase-change interfaces were susceptible to a similar treatment, particularly in view of the fact that such interfaces also arise as discontinuity surfaces in the general treatment of the equations of hydrodynamics (Keller [5]).

In the first part of this paper a formulation of certain phase transition phenomena is described (Problem II) and the concept of their weak solutions is introduced. Heuristic arguments are given serving to identify a weak solution of Problem II with the solution of Problem I. The results of several calculations are cited to lend support to a conjecture that the solution of certain natural finite difference equa-

Received February 4, 1960. A preliminary version of this paper was presented at the 13th National Meeting of the Association for Computing Machinery at Urbana, Illinois, June 13, 1958. Research was carried out under the auspices of the Atomic Energy Commission. 
tions related to Problem II converge to a relevant (essentially unique) weak solution of the problem. In addition, a random walk process suggested by the difference equation is mentioned which tends to illuminate certain aspects of phase transition processes and suggests an additional computational approach to such problems.

The author wishes to express his appreciation to P. Lax and E. Isaacson for their interest and discussions concerning this paper and to N. Metropolis for discussions of the random walk formulation.

2. Weak Solutions of Phase Transition Processes. We begin by deriving equations governing a particular example of a process involving a phase transition.* Consider [3] a bar of uniform cross-section of a substance which undergoes a change in crystalline form with negligible change in density involving a latent heat of crystallization. Let $e$ denote the specific internal energy, $q$ the heat flux, $T$ the temperature and $\rho$ the density. The equation

$$
(\rho e)_{t}+q_{x}=0
$$

expresses the conservation of energy of the process (we use the notation $v_{x}=$ $\partial v / \partial x$, etc.).

We assume Fourier's law,

$$
q=-k T_{x}
$$

where $k$ is the coefficient of thermal conductivity. Finally recalling our assumption that the density is constant, we assume an equation of state given by

$$
T=T(e) \text {. }
$$

In a region in which this transformation is one to one we obtain

$$
T_{t}=\alpha^{2} T_{x x}
$$

with $\alpha^{2}=k / \rho e_{T}$. This is the usual equation of heat conduction. However for processes in which a latent heat of recrystallization occurs the transformation (4) is no longer one to one; the following relationship is typical of such processes (see Figure 1).

$$
T=\gamma(e)+T_{0}
$$

with

$$
\gamma(e)=\left\{\begin{array}{lr}
\gamma^{-} \cdot\left(e-e_{0}\right), & e<e_{0} \\
0, & e_{0} \leqq e \leqq e_{0}+H \\
\gamma^{+} \cdot\left[e-\left(H+e_{0}\right)\right], & e>e_{0}+H .
\end{array}\right.
$$

Here $H$ is the latent heat of recrystallization and $\gamma^{-}$and $\gamma^{+}$are non-negative quantities related to the specific heat of the material. Without loss of generality we may assume $e_{0}=0$ and $T_{0}=0$.

If we adjoin to equations (2), (3) and (4') appropriate initial and boundary conditions it seems reasonable to suppose our formulation describes a unique and well-posed physical problem. Summarizing, we formulate

* Our discussion is easily generalized to several space dimensions. 


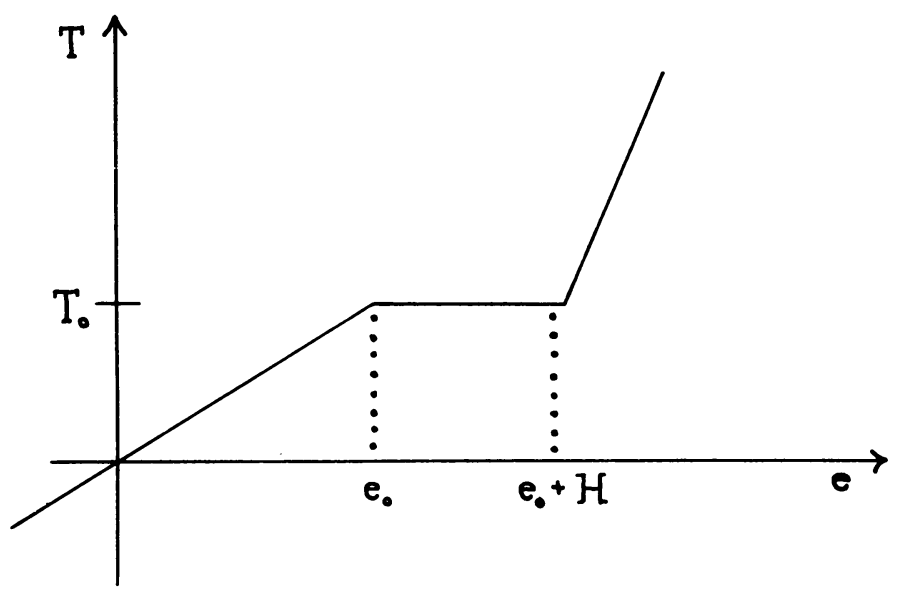

FIg. 1.-Equation of state for a recrystallization process.

Problem II: Find solutions $e(x, t), q(x, t)$ and $T(x, t)$ of the equations

$$
\begin{array}{rr}
(\rho e)_{t}+q_{x}=0, & x>0, t>0, \\
q+k T_{x}=0, & x>0, t>0, \\
T=\left\{\begin{array}{lr}
\gamma^{-} e, & e<0 \\
0, & 0 \leqq e \leqq H \\
\gamma^{+}(e-H), & e>H
\end{array}\right.
\end{array}
$$

satisfying the initial and boundary conditions

$$
\begin{array}{rlrl}
e(x, 0) & =e_{0}(x), & & x>0 \\
q(0, t) & =g(t), & t>0
\end{array}
$$

Because of the mild nonlinearity introduced by equation (4") our formulation of Problem II may not allow a genuine solution, i.e., a solution which is continuous with continuous derivatives in the quarter plane $D[x>0, t>0]$ for a compact set of initial and boundary data. The physical situation suggests that the class of solutions be enlarged to allow jump discontinuities along certain smooth curves $C$ in $D$.

We do this by introducing in the usual manner the concept of a weak solution of Problem II. Consider a space $\Phi$ of pairs of smooth testing functions $(\varphi, \psi)$ with compact support, i.e., each $(\varphi, \psi)$ in $\Phi$ vanishes identically outside some compact bounded region which lies in the half space $t>0$ and does not intersect the boundary $x=0$. We multiply equation (2) by $\varphi$ and equation (3) by $\psi$, integrate and then integrate by parts to obtain

$$
\iint\left(\varphi_{t} e+\varphi_{x} q\right) d x d t+\int \varphi(x, 0) e_{0}(x) d x=0
$$

and

$$
\iint\left(q \psi-k T \psi_{x}\right) d x d t=0
$$


recalling that $e(x, 0)=e_{0}(x)$. We restrict $(e, q, T)$ to the class of functions satisfying the boundary condition (6) and the equation of state $\left(4^{\prime \prime}\right)$ and call $(e, q, T)$ a weak solution of Problem II if $(7)$ and (8) hold for every set of test vectors $(\varphi, \psi)$ in $\Phi$.

A genuine solution is, clearly, a weak solution. On the other hand, two genuine solutions whose domains of definition are separated by a smooth curve $C$ given by $x=x(t)$ will constitute a weak solution if and only if the following equations (obtained by applying Green's theorem) hold:

$$
[\rho e] \dot{x}=[G]
$$

and

$$
[T] \dot{x}=0
$$

where $[v]$ denotes the jump in the quantity $v$ across $C$ and $\dot{x}={ }_{x} d x / d t$. Using (3), $\left(4^{\prime \prime}\right)$ and the assumption that $\rho$ is constant we obtain

$$
\rho H \dot{x}=-\left[k T_{x}\right]
$$

and

$$
[T]=0
$$

which express the conditions of balance of heat flux and the continuity of temperature across the interface. These conditions correspond to the conditions expressed in equations (1c) and (1d) of Problem I; they result in Problem II as a requirement for a weak solution.

I conjecture that a weak solution of Problem II exists which is composed of piecewise genuine solutions; furthermore it is the only such solution which is continuously dependent on the initial conditions with respect to some appropriate norm. If this conjecture can be proved correct, the identification of this solution with the solution of the Stefan problem, Problem I, would follow directly through proper choice of the initial and boundary conditions. Probably the solution of Problem II can be obtained as the limit of a certain set of finite difference equations which are described below. More precisely,

Conjecture A: There is a unique weak solution $(e, q, T)$ of Problem II composed of genuine solutions in each of the regions separated by the interface curve $x=x(t)$; furthermore, the solution $u$ of Problem I and the solution $(e, q, T)$ of Problem II (with suitable initial and boundary conditions) as identified through the relationship $T(e)=u$ are identical.

Conjecture B: $(e, q, T)$ may be obtained as the strong limit of the sequence of solutions of the finite difference equations described below (Problem $\mathrm{II}_{\Delta}$ ).

I wish to give certain plausibility arguments which, together with the results of certain calculations described later, tend to support these conjectures. In doing so I lean heavily on the already cited paper by Lax [7].

The following describes the simplest finite difference approximant to Problem II: at a point $(i, n), i=1,2, \cdots, n=1,2, \cdots$, of a mesh $\Delta$ of size $(\Delta x, \Delta t)$ replace $e_{t}$ by $\left(e_{i}{ }^{n+1}-e_{i}{ }^{n}\right) / \Delta t, q_{x}$ by $\left(q_{i}{ }^{n}-q_{i-1}^{n}\right) / \Delta x$, and $T_{x}$ by $\left(T_{i+1}^{n}-T_{i}{ }^{n}\right) / \Delta x$. This leads to 
Problem $\mathrm{II}_{\Delta}$ : Find solutions $e_{i}{ }^{n}, q_{i}{ }^{n}$, and $T_{i}{ }^{n}$ of the equations

$$
\begin{aligned}
& e_{i}^{n+1}=e_{i}{ }^{n}-\mu\left(q_{i}{ }^{n}-q_{i-1}{ }^{n}\right) \\
& q_{i}{ }^{n}=-\bar{k}\left(T^{n}{ }^{n}-T_{\imath}{ }^{n}\right) \\
& T_{i}{ }^{n}=\left\{\begin{array}{lr}
\gamma^{-} e_{i}{ }^{n}, & e_{i}{ }^{n}<0 \\
0, & 0 \leqq e_{i}{ }^{n} \leqq H \\
\gamma^{+}\left(e_{i}{ }^{n}-H\right), & e_{i}{ }^{n}>H
\end{array}\right.
\end{aligned}
$$

satisfying the initial and boundary conditions

$$
\begin{gathered}
e_{i}{ }^{0}=e_{0}(i \Delta x) \equiv e_{0 i} \\
q_{0}{ }^{n}=g(n \Delta t) \equiv g^{n}
\end{gathered}
$$

Here, $\mu=\Delta t / \rho \Delta x$ and $\bar{k}=k / \Delta x$.

Let us suppose initial and boundary conditions for which $e_{0 i} \geqq 0$ and $g^{n} \geqq 0$. It is not difficult to show then that $e_{i}{ }^{n} \geqq 0$ and the above equations can be simplified. Letting

$$
p_{i}{ }^{n}=\left\{\begin{array}{lll}
0 & \text { if } & 0 \leqq e_{i}{ }^{n} \leqq H \\
\gamma^{+} \mu \bar{k} & \text { if } \quad e_{i}{ }^{n}>H
\end{array}\right.
$$

and eliminating $q_{i}{ }^{n}$ and $T_{i}{ }^{n}$ in $\left(2_{\Delta}\right),\left(3_{\Delta}\right)$ we obtain

$$
\left(e_{i}^{n+1}-H\right)=p_{i+1}^{n}\left(e_{i+1}^{n}-H\right)+p_{i-1}^{n}\left(e_{i-1}^{n}-H\right)
$$

$$
+\left(1-2{p_{i}}^{n}\right)\left(e_{i}{ }^{n}-H\right)
$$

for $n \geqq 0, i \geqq 2$, and

$$
\left(e_{i}^{n+1}-H\right)=p_{2}{ }^{n}\left(e_{2}{ }^{n}-H\right)+\left(1-{p_{1}}^{n}\right)\left(e_{1}{ }^{n}-H\right)+\mu \bar{k} g^{n}
$$

for $n \geqq 0$.

Very likely the stability and convergence of this system is insured by requiring that the coefficients $\left(1-2 p_{i}{ }^{n}\right)$ be non-negative. This leads to the condition

$$
\frac{\Delta t}{\Delta x^{2}} \leqq \frac{\rho}{2 k \gamma^{+}}
$$

From the definition of ${p_{i}}^{n}$ it is easily seen from (11) that, if $e_{i+1}^{n}, e_{i}{ }^{n}$, and $e_{i-1}^{n}$ are each in the interval $[0, H]$, then $e_{i}^{n+1}=e_{i}{ }^{n}$. Suppose, for example, that initially $e_{0 i}=0$. We may conclude that the width of the transition region between phases, i.e., the region in which $e_{i}{ }^{n}$ varies from its initial value 0 to a value greater than $H$, is $2 \Delta x$ for any line $t_{n}=$ constant. This property provides a striking contrast to the spreading of shock zones in Lax's treatment of hydrodynamic problems and suggests that phase transitions can be determined accurately by this method.

It is of interest to consider the interpretation of equation (11) as a random walk process. Consider wells each capable of holding $H$ balls at points $P_{0}, P_{1}, \ldots$ situated on a line. A ball in a well which is not yet filled is required to remain in the well $\left(p_{i}{ }^{n}=0\right)$; a ball at a point at which the well is already filled moves to the right or left with equal probability ${p_{i}}^{n}>0$ or remains at this point with probability $\left(1-2 p_{i}{ }^{n}\right)$.

The boundary condition $q_{0}{ }^{n}$ describes the rate at which balls enter the process 
at the left and the capacity of each well measures the latent heat of the process. In addition to their theoretical interest, random walk processes of this type suggest feasible computational methods for more general, but related, problems.

3. Numerical Computations. In this section the results of several calculations based on the difference scheme outlined in Problem II are described for the following particular problem:

$$
\begin{gathered}
e_{t}+q_{x}=0 \\
q+T_{x}=0 \\
T= \begin{cases}0, & 0 \leqq e \leq 1 \\
\frac{1}{2} e, & e<0\end{cases}
\end{gathered}
$$

In each case the initial and boundary data were

$$
e(x, 0)=1 \text {, }
$$

and

$$
q(0, t)=-1
$$

The interface curve $x(t)$ was determined as the distance from the origin to the first meshpoint on the line $t_{n}=$ constant for which $T=0$. For comparison (c.f. eq. (9))

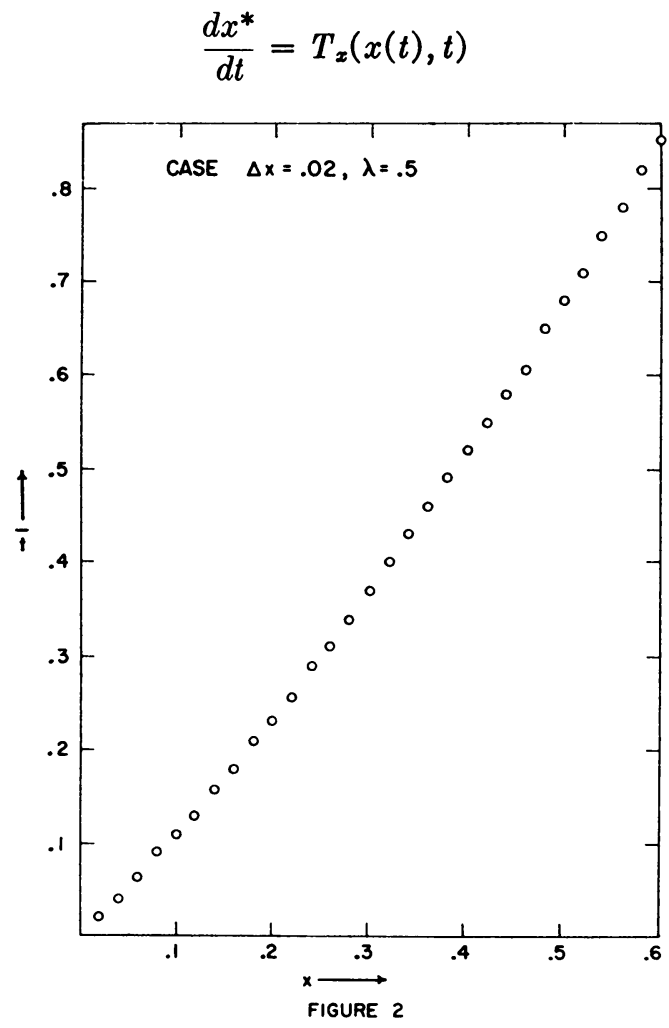

FIG. 2.-Interface for $\Delta x=.02, \lambda=.5$. 


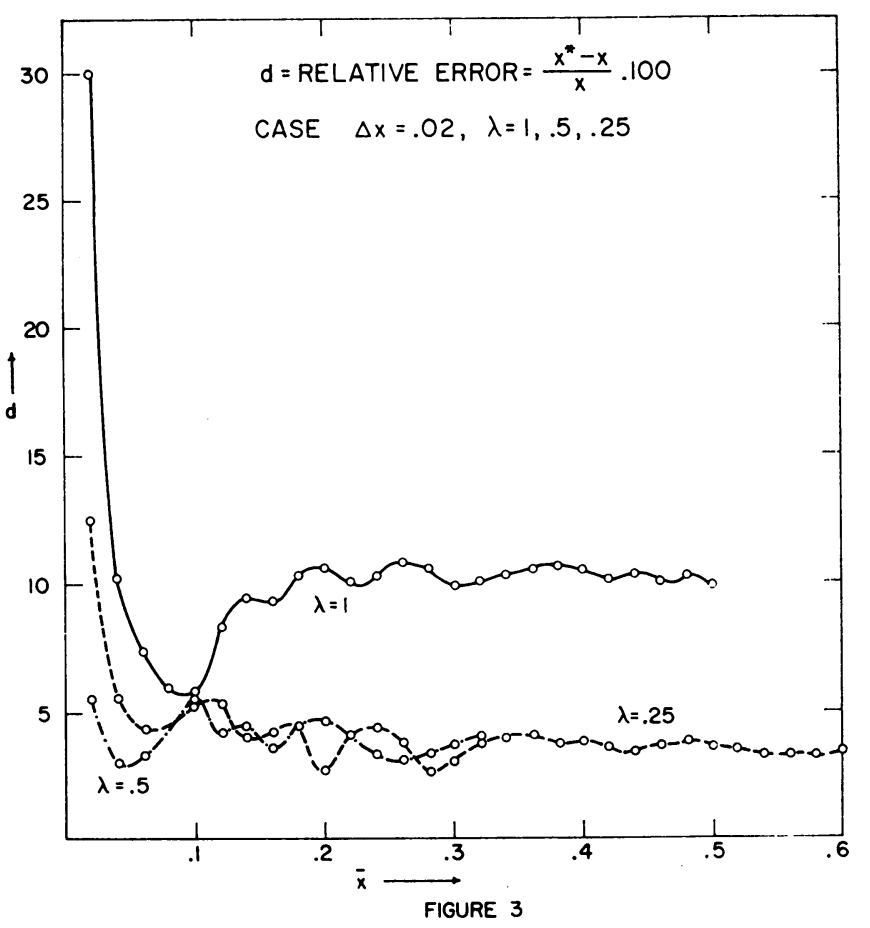

Fra. 3.-Dependence of relative error on $\lambda$.

TABLE 1

Comparison of values $t(x, \Delta x),\left(\Delta t / \Delta x^{2}=\frac{1}{2}\right)$

\begin{tabular}{c|c|c}
\hline$x$ & $t(x, .1)$ & $t(x, .02)$ \\
\hline 0.1 & 0.110 & 0.108 \\
0.2 & 0.225 & 0.232 \\
0.3 & 0.365 & 0.370 \\
0.4 & 0.515 & 0.519 \\
0.5 & 0.675 & 0.679 \\
0.6 & 0.845 & $-^{*}$ \\
0.7 & 1.025 & - $^{*}$ \\
0.8 & 1.220 & \\
\hline
\end{tabular}

* Values not calculated.

was integrated numerically using a backward difference for $T_{x}$ at $x(t)$ and the percent relative error

$$
d=100\left[\frac{x^{*}(t)-x(t)}{x(t)}\right]
$$

calculated.

Figure 2 shows the interface for the case where $\Delta x=.02$ and $\Delta t / \Delta x^{2}=.5$. Comparison to the case in which $\Delta x=.1$ and $\Delta t / \Delta x^{2}=.5$ may be made from Table 1 which compares $t(x ; \Delta x)$ for these two cases.

In Figure 3 the dependence of the relative error $d$ on the value $\lambda=\Delta t / \Delta x^{2}$ is 
given. In each case $\Delta x=.02$. As expected, instability was observed for values of $\lambda>1$, although this fact has not been indicated in the figure.

4. Conclusions. The numerical evidence presented here, although somewhat limited, seems to support the conjectures which have been described in this paper. It is unlikely that the method proposed here compares with the implicit technique of Douglas and Gallie [2] for computational efficiency, at least for one-dimensional problems. However, the present method seems well suited for computation in more dimensions. Furthermore, it is of interest to note that if, in obtaining Problem $\mathrm{II}_{\Delta}$, we were to replace $q_{x}$ by $\left[q_{i}^{n+1}-q_{i-1}^{n+1}\right] / \Delta x$, the resulting implicit difference equations would retain the feature of a sharply propagating interface and raise additional questions about the validity of our conjectures when extended to include implicit equations of this type.

Brookhaven National Laboratory

Upton, New York

1. J. CRANK, "Two methods for the numerical solution of moving-boundary problems in diffusion and heat flow," Quart. Jn. Mech. and Appl. Math., v. 10, 1957, p. 220-231.

2. J. Douglas, JR. \& T. M. Gallie, JR., "On the numerical integration of a parabolic differential equation subject to a moving boundary condition," Duke Math. Jn., v. 22, 1955, p. 557-572.

3. G. W. Evans, E. Isaacson \& J. K. L. Macdonald, "Stefan-like problems," Quart. Appl. Math., v. 8, 1950, p. 312-319.

4. A. Friedman, "Free boundary problems for parabolic equations I. Melting of solids," Jn. Math. and Mech., v. 8, 1959, p. 499-518.

5. J. B. Keller, "Geometrical acoustics I: The theory of weak shock waves," Jn. Appl. Phys., v. 25, 1954, p. 938-947.

6. I. KolODNER, "Free boundary problems for the heat equation with application to problems of change of phase," Communs. on Pure and Appl. Math., v. 9, 1956, p. 1-32.

7. P. D. LAx, "Weak solutions of nonlinear hyperbolic equations and their numerical computations," Communs. on Pure and Appl. Math., v. 7, 1954, p. 159-193.

8. W. Trench, "On an explicit method for the solution of a Stefan problem," Jn., Soc. Ind. and Appl. Math., v. 7, 1959, p. 184-204.

9. W. T. KYNER, "An existence and uniqueness theorem for a nonlinear Stefan problem," Jn. Math. and Mech., v. 8, 1959, p. 483-498. 